\title{
LA INTELIGENCIA EMOCIONAL COMO
} HABILIDAD DIRECTIVA. ESTUDIO APLICADO EN LOS MUNICIPIOS DE LA PROVINCIA DE CÓRDOBA (ESPAÑA)

\section{Emotional intelligence as a management skill. Study applied in the municipalities of the province of Córdoba (Spain)}

Francisco Javier Pereda Pérez ${ }^{1}$, Tomás López-Guzmán², Francisco González Santa Cruz ${ }^{3}$

Fecha de recepción: 12 de junio de 2016

Fecha de aceptación: 27 de octubre de 2016

1- Nacionalidad: Española. Grado: Doctorado. Especialización: Administración. Adscripción: Universidad de Córdoba. Correo: td1pepef@uco.es

2- Nacionalidad: Española. Grado: Doctorado. Especialización: Administración. Adscripción: Universidad de Córdoba. Correo: tomas.lopez@uco.es

3- Nacionalidad: Española. Grado: Doctorado. Especialización: Administración. Adscripción: Universidad de Córdoba. Correo: td1gosaf@uco.es 


\section{Resumen}

El artículo presenta una investigación sobre la inteligencia emocional como habilidad directiva en el contexto de la Administración Local en la Provincia de Córdoba (España), y en la que se posiciona como una habilidad con una gran influencia sobre el desempeño de las personas y sobre el conjunto de las variables que definen a las organizaciones municipales. A partir del trabajo de campo en diferentes entidades en el ámbito municipal, sobre un cuestionario aplicado a 362 empleados públicos, se centra en el objetivo de conocer la valoración de un inventario de 22 habilidades directivas y su importancia en las variables organizacionales. Los resultados muestran la discreta valoración otorgada a la inteligencia emocional, ofreciendo posibilidades en la definición de estrategias de formación directiva en esta habilidad, poniendo especial atención en la identificación y desarrollo del liderazgo de los directivos públicos.

Palabras clave: Inteligencia emocional, habilidades directivas, sector público, municipios, España.

\section{Abstract}

The article presents research on emotional intelligence skills policy in the context of the Local Government in the Province of Córdoba (Spain), and which is positioned as a skill with a great influence on the performance of individuals and the set of variables that define municipal organizations. From fieldwork in different entities at the municipal level, on a questionnaire given to 362 public employees, it focuses on the objective to know the valuation of inventory of 22 managerial skills and their importance in organizational variables. The results show the discrete valuation given to emotional intelligence, offering possibilities in defining strategies of management training in this skill, paying special attention to the identification and leadership development of public managers.

Keywords: Emotional intelligence, management skills, public sector, municipalities, Spain. 


\section{Introducción}

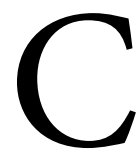

uando estudios recientes (entre otros, Goleman, 2011; Zárate y Matviuk, 2012; Del Pino Peña y Aguilar Fernández, 2013) confirman la importancia de elementos como la inteligencia emocional en el desarrollo del liderazgo, el sector público no debe quedar al margen de apostar decididamente por un elemento clave, una verdadera ventaja competitiva, que puede proporcionar un gran potencial de mejora en muchos aspectos: mejorar la motivación, la implicación y el compromiso de sus empleados, la optimización de recursos, lograr una mayor eficacia y eficiencia, etc.

La inteligencia emocional resulta ser una habilidad que ocupa cada vez un lugar más destacado y necesario en las personas, para hacer frente a las múltiples incidencias que se presentan en el desempeño laboral del día a día. Para ello, siguiendo a Goleman (1998b), se vive en una época en la que la perspectiva de futuro depende de la capacidad de controlarse a uno mismo y de manejar adecuadamente nuestras relaciones.

El desarrollo de habilidades es un tema de permanente preocupación para empresas y organizaciones, que en su afán por contar con el mejor recurso humano en los cargos de dirección y la necesidad de seleccionar profesionales capaces de tomar las decisiones adecuadas y necesarias que permitan la supervivencia de la actividad, hace que se favorezcan la generación de procesos de formación para tener una visión más estratégica, un manejo adecuado de los recursos que se encomienden y una dirección acertada del personal subordinado.

Wang y Xiao (2009), en el contexto de la auditoría de la gestión del conocimiento, valoran que las organizaciones se den cuenta de sus procesos operativos clave, tales como ganar clientes y su satisfacción, así como que los procesos que se configuran para apoyar la estrategia de la organización y poner en práctica los procesos operativos clave, destacando la gestión de recursos humanos. Es fundamental, por tanto, que la organización asuma la importancia de saber identificar y valorar adecuadamente esos procesos clave en el ámbito de la gestión de personas, entre los que destacan la identificación y desarrollo de las habilidades directivas, entre las que la inteligencia emocional ocupa un papel muy destacado. En este sentido, las prácticas de recursos humanos son el principal medio por el que las organizaciones, públicas o privadas, pueden influir en las habilidades, actitudes y comportamientos de los trabajadores y, a través de ello, en los resultados organizativos (Collins y Clark, 2003).

En este sentido, un creciente número de trabajos señalan que el uso de una serie de prácticas de recursos humanos, entre las que se encuentran procesos rigurosos de selección de trabajadores, formación y desarrollo de la carrera profesional, evaluación del rendimiento y gestión de la retribución, pueden mejorar los conocimientos y las habilidades de los empleados e incrementar su satisfacción, lo que permitirá retener a los mejores en la empresa (Sanz Valle y Sabater Sánchez, 2002; Wright, Gardner y Moynihan, 2003;Lee, Lee y Wu, 2010; Kim y Lee, 2012).

En este artículo se presenta los resultados de una investigación realizada a empleados del sector público municipal de la provincia de Córdoba (España), y que permite extraer una serie de resultados sobre la importancia de la inteligencia emocional como habilidad directiva, de los que se obtienen conclusiones en un campo de conocimiento de trascendencia en el que los procesos de cambio se hacen presentes de forma inexorable. 
La estructura de este artículo consta, en primer lugar, además de la presente introducción, de una revisión teórica sobre la habilidad inteligencia emocional, para continuar con un apartado dedicado a la descripción del área geográfica. Posteriormente se analiza la metodología aplicada y, a continuación, se presentan los principales resultados de la investigación. Se finaliza el artículo con las conclusiones más relevantes y futuras líneas de investigación, que persiguen avanzar en el conocimiento científico sobre la inteligencia emocional como habilidad directiva en el contexto del sector público municipal, así como con las referencias bibliográficas utilizadas.

\section{Revisión teórica}

El constructo Inteligencia Emocional tiene sus orígenes en los estudios de Edward Thorndike (1920) sobre la Inteligencia Social, definida como la capacidad para comprender y dirigir a las personas y gestionar sus relaciones. Más tarde, Gardner (1983) abundó en los conceptos de inteligencia interpersonal e intrapersonal señalando que la primera, la inteligencia interpersonal, denota la capacidad para comprender las intenciones, motivaciones y deseos de los demás para trabajar eficazmente con ellos y, la segunda, la inteligencia intrapersonal, como la capacidad para comprenderse a uno mismo de forma efectiva.

En cambio, no fue hasta Salovey y Mayer (1990) cuando se realizaron las primeras investigaciones relevantes sobre este concepto. Estos autores se enfocaron en componentes afectivos, emocionales, personales y sociales (sin menospreciar los cognitivos) que suponían un factor clave de éxito en los diferentes ámbitos de la vida. Sin embargo, fue Goleman (1996) quien realmente popularizó la inteligencia emocional identificando los atributos necesarios para triunfar en la vida y ser feliz. Además, explicó como la inteligencia emocional no es un factor inamovible, sin capacidad de modificación, sino que es un constructo que es posible desarrollar y potenciar a lo largo de la vida. El destacado psicólogo norteamericano Bar-On (1997) definió la inteligencia emocional como un conjunto de capacidades, competencias y habilidades no cognitivas que influencian la habilidad propia de tener éxito al afrontar las demandas y presiones del entorno.

Por su parte, Cooper y Sawaf (1998), destacan el papel que las emociones tienen en lo que mueve a cada individuo, siendo que a través de la inteligencia emocional se aprende a reconocerlas, valorarlas en sí mismos y en los demás, de manera que se pueda responder adecuadamente a ellas, aplicando eficazmente la energía de las emociones en la vida diaria y en el trabajo.

Dolan (2005) considera que una empresa que cuenta con profesionales de alta calidad técnica y humana posee una herramienta importante para la gestión empresarial, siendo dicha herramienta la que puede marcar la diferencia en el camino al éxito entre las empresas porque la ventaja competitiva puede encontrarse en la habilidad de transmitir y adaptar el know-how único y específico de conocimientos tecnológicos, estratégicos o comerciales de manera eficaz a otras realidades. Esa herramienta a la que se refería Dolan es la inteligencia emocional, constructo que a pesar de que su aparición en la literatura científica es relativamente reciente (finales del siglo XX), su importancia y relevancia actual ha superado notablemente todas las expectativas.

Por su parte, Goleman (1996) define el término inteligencia emocional como la capacidad humana de sentir, entender, controlar y modificar estados emocionales en uno mismo y en los demás. Inteligencia emocional no es ahogar las emociones, sino dirigirlas y equilibrarlas. Las emociones, esas grandes condicionantes de la conducta, pueden influenciar la vida en multitud de sentidos. El mismo Goleman (1998a) 
completa la definición anterior afirmando que la inteligencia emocional es la capacidad de motivarse a uno mismo, de perseverar en el empeño a pesar de las posibles frustraciones, de controlar los impulsos, de diferir las gratificaciones, de regular los propios estados de ánimo, de evitar que la angustia interfiera con las facultades racionales.

Madrigal (2009) se pregunta por qué la importancia de la inteligencia emocional en el directivo es prioritaria en toda organización. La respuesta muestra la relevancia de esta habilidad imprescindible, pues de ello depende la empresa y la organización, así como la emoción, la pasión, el énfasis y el sentimiento positivo o negativo con que trabaje el directivo y, por ende, su equipo de trabajo o colaboradores. López-Boudet y Martínez-Vázquez (2014) consideran que desde la aparición de los estudios sobre inteligencia emocional se ha producido un verdadero movimiento de aplicación de ese tipo de inteligencia para el análisis y la evaluación del desempeño de personas e instituciones.

Efectivamente, esta megahabilidad, pues como se verá más adelante engloba una serie de componentes que se identifican en la Figura 1, está teniendo influencia en las empresas que se acercan a este constructo, y un gran interés por descubrir las potencialidades que ofrece. Para ello hace falta una alfabetización emocional que permita que la gestión de personas en las organizaciones se desarrolle por parámetros nuevos.

Figura1. Componentes de la inteligencia emocional

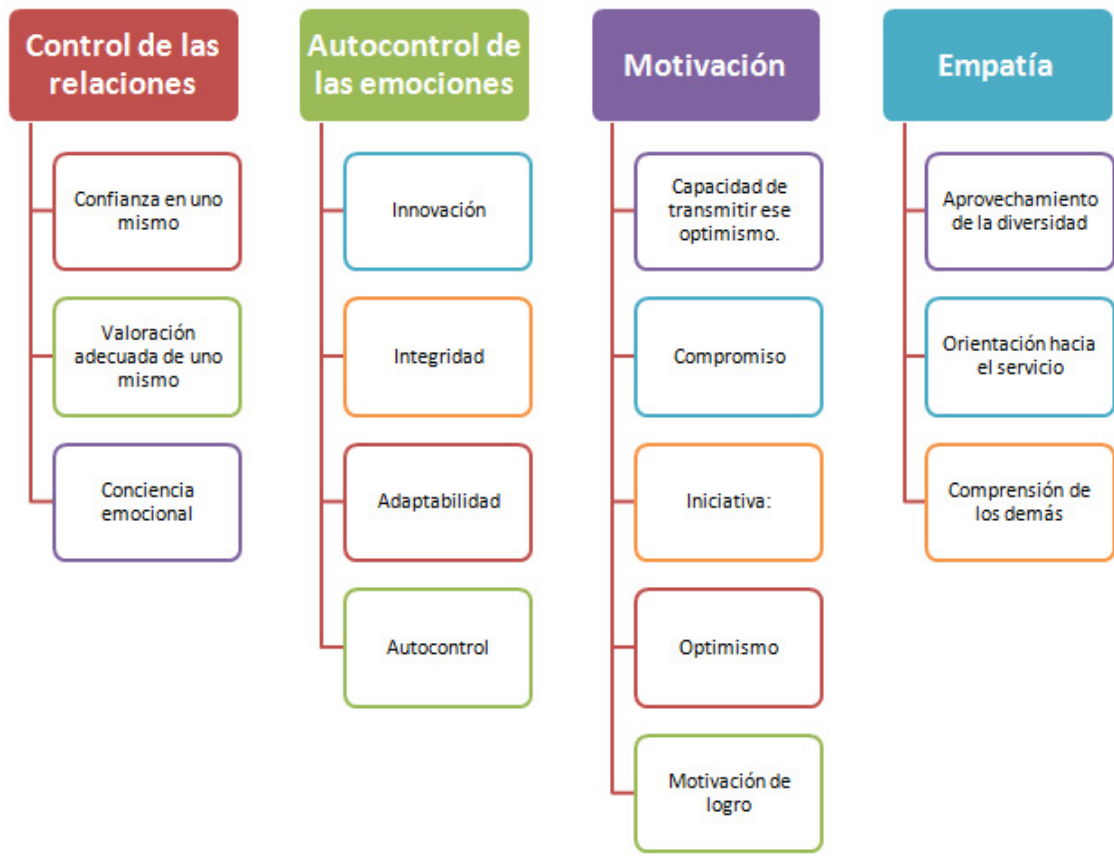

Fuente: Elaboración propia a partir de Goleman (1996)

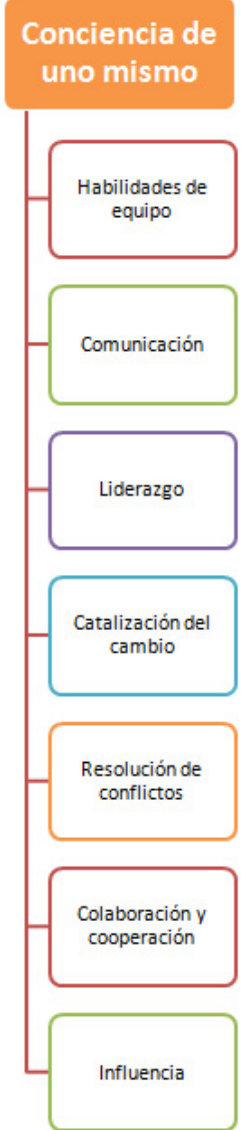


En la presente investigación se ha utilizado la siguiente definición de inteligencia emocional: "habilidad para reconocer y procesar la información que transmiten las emociones y sus relaciones con el entorno, para razonar y resolver problemas eficazmente" (Salovey y Mayer, 1990: 189). Para su estudio y análisis, la inteligencia emocional incluye dos tipos de inteligencias: la inteligencia personal y la inteligencia interpersonal. En conjunto, comprende un conjunto de destrezas, actitudes, habilidades y competencias que determinan la conducta de un individuo, sus reacciones, estados mentales, etc.

La inteligencia personal está integrada por una serie de competencias que determinan el modo en que las personas se relacionan con uno mismo. Cuando se aplica en el trabajo, comprende tres componentes: la automotivación, la autoconciencia y el autocontrol (Figura 2). Respecto a la inteligencia interpersonal, se destaca la capacidad de reconocer las emociones de los demás, que implica la empatía, la cual se basa en el conocimiento de las propias emociones. Otro elemento fundamental es la capacidad de establecer relaciones, que en gran medida supone la habilidad de manejar las propias emociones. La competencia social y las habilidades que conlleva son la base del liderazgo y eficiencia interpersonal. Las personas que dominan estas habilidades sociales son capaces de interactuar de forma efectiva con los demás (Bisquerra, 2003).

Figura2. Componentes de la inteligencia personal

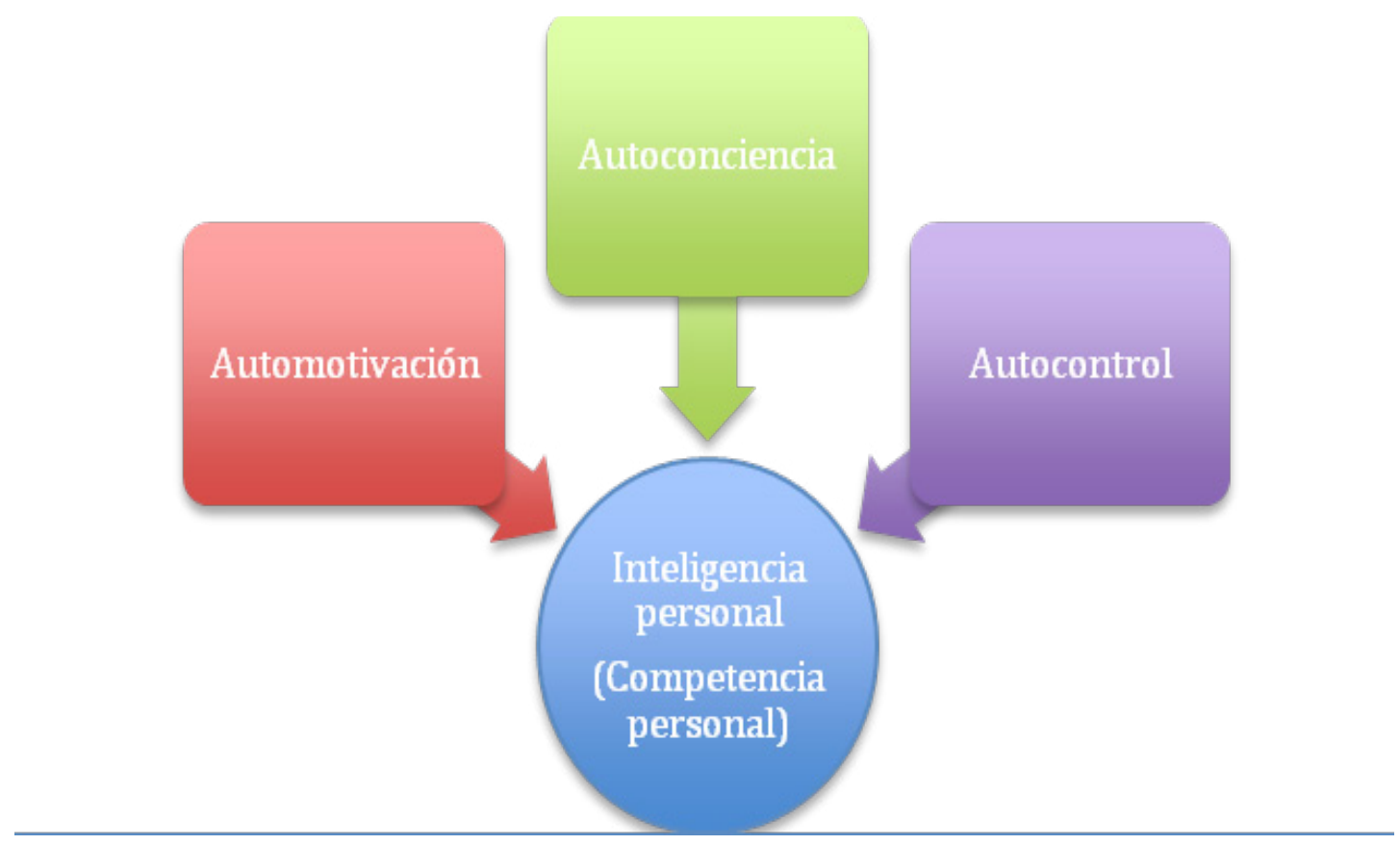

Fuente: Elaboración propia a partir de Goleman (1996)

Ser inteligente emocionalmente, supone poner el acento en otros aspectos que hasta hace unos años, se consideraban competencias blandas (soft), que se identifican con las habilidades. En las organizaciones ha primado las competencias duras (hard) relativas al conocimiento sobre el puesto de trabajo, la técnica, etc. Ha sido más que evidente que se ha priorizado y conducido la orientación 
profesional y personal de los individuos en función casi siempre del conocimiento, soslayando otras. Afortunadamente desde el desarrollo de las teorías de inteligencias múltiples ha favorecido que la educación y la formación de las personas puedan ir encaminada al desarrollo de habilidades clave para el éxito en la vida. Ese es el gran logro de estas teorías, poner el foco de atención en pro de la felicidad y el éxito, más en habilidades intra e interpersonales que en el cociente intelectual de cada uno.

Las numerosas investigaciones que se han generalizado a partir de haberse popularizado este constructo han permitido avanzar en el conocimiento de un aspecto fundamental en las empresas y organizaciones en general. Por ejemplo, Batista y Bermúdez (2009) demuestran la correlación positiva entre las variables inteligencia emocional y el liderazgo femenino en cargos de dirección en organismos públicos. Dentro de las actividades públicas, Hopkins, O’Neil y Williams (2007) encontraron que la inteligencia emocional es un factor crítico para el liderazgo eficaz entre los consejos escolares. Iguales conclusiones aportan Alegre y Levitt (2014) sobre la estrecha relación entre la inteligencia emocional y el liderazgo transformacional, aunque aportan como novedad que se obtienen resultados contradictorios en función de las diferentes definiciones de inteligencia emocional utilizadas en las investigaciones.

Blais y Sinclair-Desgagné (2002) consideran que la investigación sobre las competencias de liderazgo ha subrayado la contribución significativa de lo que ahora se llama inteligencia emocional. Estos autores resaltan que los principales ingredientes del liderazgo serían la autoconciencia y la empatía, esta última abarca la capacidad de escuchar a los demás y la integración de sus respectivos puntos de vista. Sobre este mismo asunto, la efectividad del liderazgo, Guillén y Florent-Treacy (2011) indican que la inteligencia emocional tiene un efecto significativo en los comportamientos de colaboración en el trabajo.

Serrano y Barba (2012) centran su investigación en la necesidad de formación, que en el caso de la Administración Pública, debe tener como objetivo adaptarse a las necesidades cambiantes de los ciudadanos a los que se prestan los servicios, manteniendo actualizados los conocimientos y las habilidades del personal. En este sentido, de acuerdo con el estudio de casos realizado por Coggburn y Hays (2004), aquellos ayuntamientos que muestran un fuerte compromiso con la formación consiguen mejorar la satisfacción en el trabajo y aumentar la calidad de vida de la mayoría de sus empleados públicos. Una vez más, la importancia del diseño del plan de formación partiendo de un correcto análisis de necesidades y teniendo en cuenta las habilidades esenciales que deben desempeñarse en cada puesto de trabajo resulta determinante para obtener los mejores resultados.

En España, la investigación de Cardona y Chinchilla (1999) señalan que las competencias directivas son una herramienta esencial para asegurar la competitividad de las empresas en la nueva economía global; definen las habilidades como aquellas capacidades operativas que facilitan la acción. El desarrollo de las mismas requiere un proceso de entrenamiento, y con la repetición de las acciones se adquieren nuevos hábitos y modos efectivos para actuar. Consideran a la inteligencia emocional como una competencia de eficacia personal, que son aquellos hábitos que facilitan una relación eficaz de la persona con su entorno.

En esta línea argumental, resulta interesante el trabajo de Ryback (2005), que presenta lo que denomina "Los diez atributos de la inteligencia emocional ejecutiva", que se muestran en el Cuadro 1. 


\section{Cuadro 1. Los diez atributos de la Inteligencia Emocional Ejecutiva}

Actitud libre de juicios de valor: Sacar lo mejor de los demás: aceptar a cada individuo sobre la base de lo que éste ofrece en el momento presente, y no en función de lo que piensen los demás que pueda haber sucedido en el pasado.

Perceptividad: Ayudar a los demás a que se comprendan a sí mismos: comprender a los demás y, con la sabiduría que les otorga su experiencia, les ayudan a comprenderse a si mismos, haciendo que se sientan valorados.

Sinceridad: Fomentar la honradez genuina: se refiere a expresar con honradez los sentimientos e intenciones propios.

Presencia: Asumir la responsabilidad personal: apertura ante hechos o sentimientos que se presenten en un momento dado, ocuparse personalmente de los problemas propios de sus responsabilidades y establecer un contacto directo con los más afectados.

Relevancia: Apoyar la verdad: capacidad de abordar el qué, el cuándo, el quién, y el cómo de cualquier situación: Los datos concretos que describen los objetivos, los problemas y soluciones.

Expresividad: Ser abierto y directo, aunque con sentido claro de la oportunidad, midiendo constantemente el efecto que tiene la expresión personal sobre el público. Animar a los demás a alcanzar niveles más elevados de energía y dedicación.

Apoyo a los demás: Fomentar la lealtad y un sentimiento de aportar algo. Hacer que las personas sientan que son algo más que adecuadas, que son singulares y que están realizando un aporte significativo a la causa de la empresa.

Audacia: Resolver pronto los conflictos: asumir la iniciativa de hacer frente a las discrepancias antes de que los otros las hayan observado, escuchar sin prejuicios los datos relevantes. Reaccionar con calma antes los intentos de engaño y evasivas.

Celo: Presentar un modelo de liderazgo efectivo: Sentirse intensamente involucrados a lo largo del día y sensibles, tanto a sus propios sentimientos como a los de los que nos rodean.

Confianza en sí mismo: Facilitar que los demás se sientan tranquilos y seguros en sus roles, animándoles para que profundicen en sus recursos personales para llevar a cabo su tarea.

Fuente: Ryback (2005)

La relación entre la inteligencia emocional y la actividad laboral ha sido confirmada por numerosos estudios (Sternberg, 1997; Ryback, 1998; Weisinger, 2001). El conocimiento de la inteligencia emocional de un directivo es importante, ya que de él depende el trabajo de otras personas, que se ven influenciadas por el despliegue de su inteligencia emocional, afectando esto al conjunto de la organización. Las organizaciones afrontarán mejor las exigencias de un entorno tan exigente y cambiante si están integradas por directivos y trabajadores con inteligencia emocional, capaces de hacer frente a cambios y desempeñar sus responsabilidades con mayor eficacia. Son directivos que valoran el trabajo en equipo, la cooperación con otras personas, la capacidad de adaptación y la orientación al logro (Goleman y Cherniss, 2005). 
Desde esta perspectiva, Goleman destaca que "La aptitud emocional es importante sobre todo en el liderazgo, papel cuya esencia es lograr que otros ejecuten sus respectivos trabajos con más efectividad. La ineptitud de los líderes reduce el desempeño de todos: hace que se malgaste el tiempo, crea asperezas, corroe la motivación y la dedicación al trabajo, acumula hostilidad y apatía" (Goleman, 1998c: 52). El directivo debe ser capaz de manejar situaciones complejas para optimizar sus resultados a partir del conocimiento profundo de la naturaleza humana, sus expectativas, necesidades y motivaciones, para lo cual es necesario, capacidad para comprender (empatía), saber generar confianza entre las personas que dirige (desarrollo de personas), saber escuchar, comunicar de forma convincente, generando ilusión, entusiasmo y compromiso en las personas, a través de objetivos y proyectos ilusionantes.

\section{Descripción de la zona geográfica}

La investigación se ha desarrollado en la provincia de Córdoba, que ocupa una extensión de 13.770 $\mathrm{km} 2$, situada en el centro de la región de Andalucía (España). Tiene una población de 795.611 habitantes (dato a 1 de enero de 2015) (Instituto Nacional de Estadística, 2016a), que representa el 9,56\% de la población andaluza y el 1,72\% de la población española. La capital de la provincia está situada en la ciudad de Córdoba, que reúne al 40,96\% de toda la población (Figura 3).

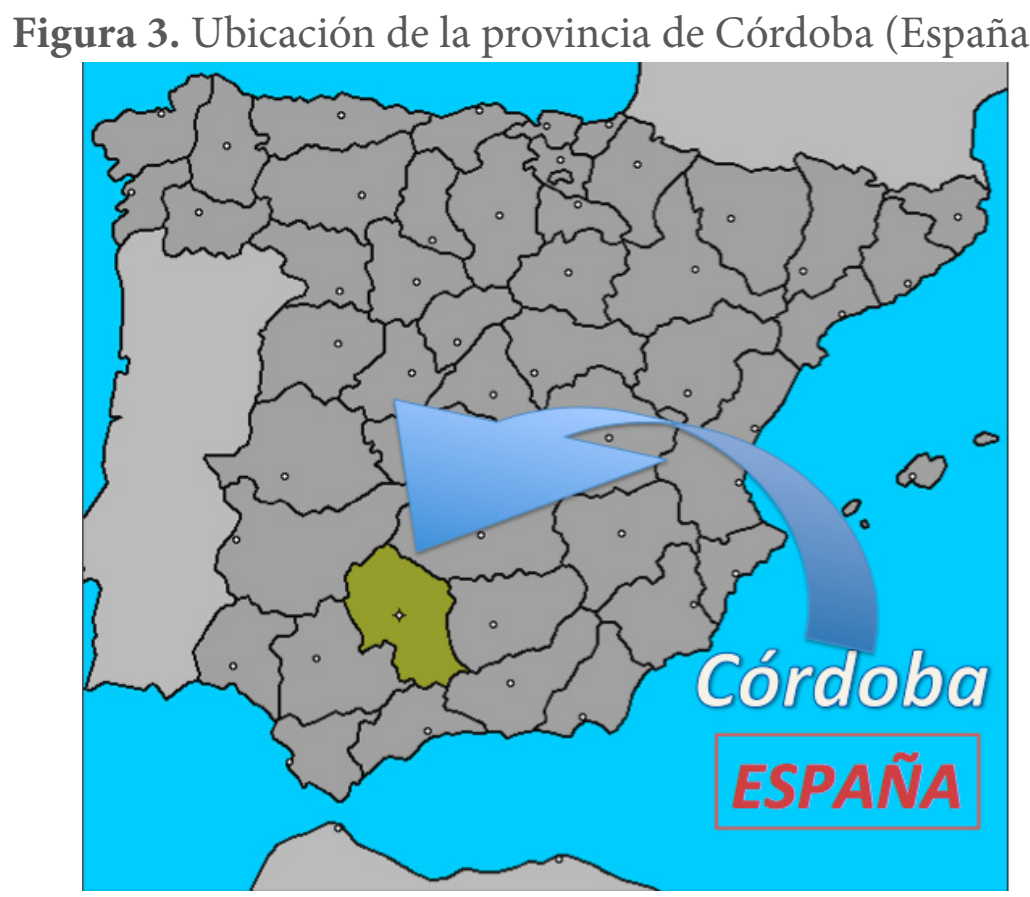

Fuente: Elaboración propia a partir de

http://www.luventicus.org/mapas/espanaprovincias/cordoba.html

La provincia de Córdoba se organiza administrativamente en torno a 75 municipios, entre los que predominan los de menos de 5.000 habitantes el 62,7\%, si bien representan el 13,5\% de la población total 
de la provincia. En cambio, ocho municipios, entre ellos la capital, suponen el 63,7\% de la población total. Respecto a la población, se distribuye por sexos con un $49,12 \%$ de hombres y un $50,88 \%$ de mujeres.

El análisis de los sectores económicos en la provincia de Córdoba muestra una excesiva concentración en el sector servicios, que llega a representar el 67\% del PIB provincial. Dentro de este sector servicios destaca de forma clara el turismo, aunque el sector agrícola, que antaño llego a tener una gran importancia, ha visto cómo iba perdiendo importancia relativa paulatinamente, a la vez que la ganaba el sector servicios, resultado del estudiado proceso de desagrarización y terciarización de la economía española iniciado en los años 60 del pasado siglo. Hay que destacar que esta importancia del sector servicios tiene relación con el peso específico que el sector público representa, con una amplia tipología de entidades que ofrecen numerosas actividades de gran relevancia para los ciudadanos.

Respecto al número de empleados públicos ocupados en la Administración Local, a 1 de enero de 2015 se situaba en 12.777 empleados públicos (Ministerio de Hacienda y Administraciones Públicas, 2015), entre personal laboral y personal funcionario, distribuidos entre un importante número de entidades de diferente personalidad jurídica (Cuadro 2). Este dato, en relación con años precedentes viene a constatar que el ajuste general que se está produciendo en los recursos humanos del sector público en los últimos años (debido a la grave crisis económica que está sufriendo España), en el ámbito de la administración local ha sido inexistente, con niveles de efectivos incluso superiores a los de años previos a la crisis económica (11.457 empleados en 2011 y 10.950 en 2007).

\section{Cuadro 2. Empleados públicos}

\begin{tabular}{|c|c|c|}
\hline ÁMBITO & ADMINISTRACIÓN LOCAL & TOTAL \\
\hline ESPAÑA & 577.164 & 2.544 .804 \\
\hline ANDALUCIA & 138.778 & 487.228 \\
\hline CÓRDOBA & 12.777 & 46.175 \\
\hline
\end{tabular}

Fuente: Ministerio de Hacienda y Administraciones Públicas (2015).

En relación a la tasa de paro, la provincia de Córdoba muestra una situación claramente más desfavorable respecto a la media española, con casi nueve puntos de diferencia, concretamente un 20,5\% en España frente al 29,7\% en Córdoba (Instituto Nacional de Estadística, 2016b). En general, la asombrosa capacidad de la economía española para destruir empleo en los periodos de crisis económica, contrasta con una limitada fortaleza a la hora de crear puestos de trabajo cuando las circunstancias se tornan más favorables. Respecto a la internacionalización de la economía cordobesa, componente que ha ocupado un papel destacadísimo durante la crisis económica, constituyéndose una verdadera salida a muchas empresas que percibían cómo la demanda interna no ofrecía posibilidades de crecimiento, ocupa un nivel intermedio a nivel regional andaluz. 


\section{Metodología}

La investigación objeto de este trabajo se llevó a cabo mediante un estudio empírico centrado en la Administración Local de la Provincia de Córdoba, que incluía a una importante representación de Ayuntamientos y otras entidades dependientes de los mismos y dentro de éstas, la población universo se refería a todos los empleados públicos, funcionarios y laborales.

\section{Cuadro 3. Ficha técnica de la investigación}

\begin{tabular}{|c|c|}
\hline Ámbito geográfico: & Provincia de Córdoba (España) \\
\hline Universo: & Personal de la Administración Local \\
\hline Tamaño del universo: & 12.777 empleados \\
\hline Modo de aplicación: & $\begin{array}{c}\text { Presentación personal y cuestionario estructurado y cerrado y } \\
\text { mediante cuestionario online. }\end{array}$ \\
\hline Tamaño de la muestra: & 362 cuestionarios \\
\hline Error muestral: & $5,01 \%$ \\
\hline Período de recogida de información: & Septiembre-diciembre 2013 \\
\hline
\end{tabular}

Fuente: Elaboración propia

El instrumento para la recogida de la información ha consistido en un cuestionario estructurado y cerrado, en soporte papel y online, constituido por cuatro bloques (adaptado de (González, Sánchez y López, 2013): Bloque I: Variables sobre la Administración a la que pertenece. Bloque II: Variables relacionadas con el puesto de trabajo, basado en el cuestionario de Robina (2002). Bloque III: Variables relacionadas con las habilidades directivas en el desempeño de su puesto de trabajo. Bloque IV: Variables sociodemográficas.

El trabajo de campo se realizó durante los meses de septiembre a diciembre del año 2013, comenzando con la identificación de las entidades locales de la provincia de Córdoba (clasificadas en una diversa tipología de entidades: Ayuntamientos, Organismos Autónomos, Mancomunidades y Consorcios, Empresas públicas). A partir de una primera toma de contacto, telefónica o personal, a los responsables de las diferentes entidades presentándoles el proyecto de investigación, se solicitaba su colaboración y concertaba una reunión para explicarles in situ los objetivos principales perseguidos, realizando de manera simultánea la entrega de las correspondientes encuestas y sobres en blanco.

Se ha considerado más apropiada la encuesta escrita sobre otras posibilidades como la entrevista personal, la encuesta telefónica, etc., en virtud de algunas de las ventajas que presenta, tales como su reducido coste de realización, el alto grado de accesibilidad a la población objetivo y la probabilidad inferior de errores de trascripción en el tratamiento de datos. 
Ente las diversas partes del cuestionario, destaca a efectos del presente artículo, el apartado referente al "Bloque III: Variables relacionadas con las habilidades directivas en el desempeño de su puesto de trabajo", y tiene su fundamento científico en el "Cuestionario de Competencias Directivas" (GarcíaLombardía, Cardona y Chinchilla, 2001), sobre las competencias directivas más valoradas. Se trata de un cuestionario fácil de administrar y que se puede usar en diferentes organizaciones entre una gran variedad de empleados.

La amplia utilización de este instrumento entre la comunidad científica que ha estudiado el liderazgo y las habilidades directivas en general, tiene su base principal en sus buenas propiedades psicométricas. La valoración de 1 a 5 según una escala de Likert ofrecía una gran cantidad de información. En el cuestionario se presentan un total de treinta competencias con su definición correspondiente. De esta manera se consigue unificar el sentido de cada una de estas competencias, de forma que se asegure que se está valorando una competencia concreta por la que se pregunta.

En la investigación se ha utilizado el programa SPSS versión 21, realizándose un análisis estadístico que permite definir el perfil del empleado público en la provincia de Córdoba, desde la perspectiva de las habilidades directivas. La utilización del cuestionario en formato papel y telemático, se realizó en función de la dispersión de la plantilla en más de un centro de trabajo, así como en el grado de utilización e implantación de las nuevas tecnologías. No obstante, la inmensa mayoría sigue optando por la encuesta en formato papel, posiblemente, por la desconfianza a suministrar información personal a través de medios electrónicos o digitales (89,23\% en formato papel y 10,77\% en formato telemático).

En resumen, la muestra seleccionada alcanzó un tamaño muestral de 381 encuestas, de las cuáles 362 fueron válidas, lo que representa un error muestral del 5,01\%. En total se seleccionaron 26 entidades y unidades administrativas diferentes pertenecientes, tanto a servicios centrales como a servicios periféricos del ámbito local, elegidas específicamente con objeto de abarcar entidades y actividades diferentes (administración general de los ayuntamientos, policía local, urbanismo, gestión informática, saneamiento y medio ambiente, suministro de agua potable, etc.), localización geográfica dispar (en la ciudad de Córdoba y en municipios de la provincia, de mayor y menor población) y dimensiones distintas (desde pequeñas organizaciones hasta macro entidades de más de 1.000 empleados públicos).

\section{Resultados}

La presente sección está dedicada a exponer los resultados obtenidos en la investigación realizada, en el que se incluye una amplia y completa descripción de las variables analizadas, realizando tanto un análisis univariante como bivariante. Se presentan, en primer lugar, el análisis descriptivo de las principales variables consideradas: aspectos sociodemográficos, aspectos profesionales, factores de ingreso en la administración, aspectos relacionados con el desempeño del puesto. En segundo lugar se presenta con la valoración de las habilidades, tanto a nivel de responsables como de subordinados, haciendo especial énfasis en la habilidad inteligencia emocional. También se aborda el análisis sobre la valoración de la influencia de las habilidades sobre variables organizacionales. En el Cuadro 4 se recoge el perfil sociodemográfico y profesional de los empleados públicos de la Administración Local de la provincia de Córdoba. 
Cuadro 4. Perfil sociodemográfico del empleado público local

\begin{tabular}{|c|c|c|c|c|c|}
\hline Variables & & $\%$ & Variables & $\mathbf{N}^{\circ}$ & $\%$ \\
\hline \multicolumn{3}{|l|}{ Sexo } & \multicolumn{3}{|l|}{ Estado civil } \\
\hline Mujer & \multirow{2}{*}{$N=361$} & 42,9 & Soltero/a & \multirow{4}{*}{$\mathrm{N}=362$} & 7,5 \\
\hline Hombre & & 57,1 & Casado/a & & 85,3 \\
\hline \multicolumn{3}{|c|}{ Edad (años) } & Divorciado/a & & 6,6 \\
\hline De 18 a 25 años & \multirow{6}{*}{$\mathrm{N}=363$} & 0 & Viudo/a & & 0,6 \\
\hline De 26 a 35 años & & 7,4 & \multicolumn{3}{|c|}{ Nivel de formación } \\
\hline De 36 a 45 años & & 34,7 & \multirow{2}{*}{ Escuela primaria y secundaria } & \multirow{5}{*}{$\mathrm{N}=361$} & \\
\hline De 46 a 55 años & & 43,3 & & & 39,9 \\
\hline De 56 a 65 años & & 14,3 & \multirow{2}{*}{ Educación universitaria } & & \multirow{2}{*}{53,2} \\
\hline Más de 65 años & & 0,3 & & & \\
\hline \multicolumn{3}{|c|}{ Nivel salarial en euros (Retribución neta) } & Máster o Doctorado & & 6,9 \\
\hline Hasta $1.000 € /$ mes & \multirow{6}{*}{$\mathrm{N}=363$} & 1,9 & \multicolumn{3}{|c|}{ Afiliación sindical } \\
\hline De 1.001 a $1.500 € /$ mes & & 32,8 & Si & \multirow{2}{*}{$N=361$} & 32,1 \\
\hline De 1.501 a $2.000 € /$ mes & & 34,4 & No & & 67,9 \\
\hline De 2.001 a $2.500 € /$ mes & & 17,6 & \multicolumn{3}{|c|}{ Formación específica en habilidades } \\
\hline De 2.501 a $3.000 € /$ mes & & 10,2 & Si & \multirow{2}{*}{$\mathrm{N}=362$} & 50,6 \\
\hline Más de $3.000 € /$ mes & & 3 & No & & 49,4 \\
\hline
\end{tabular}

Fuente: Elaboración propia a partir del trabajo de campo.

De acuerdo con los datos obtenidos en el cuadro anterior, se pueden obtener el perfil del empleado público de la Administración Local en la provincia de Córdoba: a) existe una mayor presencia del hombre (57,1\%); b) más de dos tercios de los empleados (78\%) tienen menos de 55 años, destacándose el intervalos 45-55 años con el 43,3\%; c) la gran mayoría están casado/a, pues representan nada menos que el 85,4\%; d) la mitad de los empleados tiene formación universitaria (53,2\%); e) el nivel salarial muestra una gran concentración $(67,2 \%)$ en los intervalos que van de 1.001 y 2.000 euros/mes; f) la importante afiliación sindical $(32,1 \%)$ y, g) es significativo el equilibrio entre los que sí han recibido formación específica $(50,6 \%)$ en materia de habilidades, frente a los no han recibido ninguna formación $(49,4 \%)$.

Respecto al perfil profesional (Cuadro 5), la radiografía obtenida responde a las siguientes características: a) se constata una gran mayoría de personal funcionario (trabajadores que tienen una relación con la Administración Local que se rigen por el Derecho Administrativo, es permanente en el tiempo y es la figura habitual que tiene asignada el ejercicio de las funciones de potestades públicas o salvaguardia de los intereses generales de la Administración) frente a personal laboral (trabajadores que tienen regulada su relación con la Administración Local mediante contrato de trabajo, el convenio colectivo y las normas laborales vigentes), concretamente un $67,3 \%$ frente a un $32,7 \%$ respectivamente; b) en cuanto al grupo de clasificación profesional actual, destacan dos grupos sobre el resto, el C1, en funcionarios, con el 37,3\% y el I, en laborales, con el 29,2\%; c) destacan entre los puestos de responsabilidad el de Jefatura de Negociado, Unidad o Grupo con el 35,7\%, por tanto, se trata de puestos intermedios, puestos que representan un papel destacadísimo en el sector público, y especialmente en la Administración Local; d) se constata 
una gran mayoría de puestos directivos (85\%) con unos efectivos a su cargo de hasta 25 trabajadores, y finalmente, e) se produce una gran mayoría de los empleados públicos trabajan en entidades que se corresponden con la administración general (75,2\%), mientras que casi un $25 \%$ trabajan en otras entidades con personalidad jurídica dispar (empresa pública, consorcio, etc.).

Cuadro 5. Perfil profesional del empleado público local

\begin{tabular}{|c|c|c|c|c|c|}
\hline \multicolumn{6}{|c|}{ Tipo de personal } \\
\hline \multicolumn{3}{|c|}{ Funcionarios } & \multirow{2}{*}{$N=346$} & \multicolumn{2}{|r|}{67,3} \\
\hline \multicolumn{3}{|l|}{ Laborales } & & \multicolumn{2}{|r|}{32,7} \\
\hline \multicolumn{3}{|c|}{ Grupo clasificación actual (Funcionarios) } & \multicolumn{3}{|c|}{ Grupo clasificación actual (Laborales) } \\
\hline Grupo A-A1 & \multirow{5}{*}{$\mathrm{N}=233$} & 24,5 & Grupo I & \multirow{5}{*}{$\mathrm{N}=113$} & 29,2 \\
\hline Grupo B-A2 & & 15 & Grupo II & & 23,9 \\
\hline Grupo C-C1 & & 37,3 & Grupo III & & 21,2 \\
\hline Grupo D-C2 & & 22,3 & Grupo IV & & 18,6 \\
\hline Grupo E-AP & & 0,9 & Grupo V & & 7,1 \\
\hline \multicolumn{3}{|c|}{ Puesto directivo } & \multicolumn{3}{|c|}{$\mathrm{N}^{\circ}$ de empleados a cargo } \\
\hline Director General & \multirow{6}{*}{$N=182$} & 2,2 & Hasta 25 & \multirow{4}{*}{$N=147$} & 85 \\
\hline Gerente & & 2,7 & De 26 a 100 & & 10,2 \\
\hline Jefe de Servicio & & 17,6 & De 101 a 250 & & 3,4 \\
\hline Jefe de Departamento & & 19,2 & De 251 a 1.000 & & 1,4 \\
\hline Jefe de Neg., Unidad o Grupo & & 35,7 & & & \\
\hline Otros & & 22,5 & & & \\
\hline \multicolumn{6}{|c|}{ Tipo de Entidad } \\
\hline \multicolumn{3}{|c|}{ Administración General } & \multirow{4}{*}{$N=367$} & \multicolumn{2}{|r|}{75,2} \\
\hline \multicolumn{3}{|c|}{ Organismo Autónomo } & & \multicolumn{2}{|r|}{7,1} \\
\hline \multicolumn{3}{|c|}{ Consorcio/Mancomunidad/Patronato } & & \multicolumn{2}{|r|}{4,6} \\
\hline \multicolumn{3}{|c|}{ Empresa pública } & & \multicolumn{2}{|r|}{13,1} \\
\hline
\end{tabular}

Fuente: Elaboración propia a partir del trabajo de campo.

Seguidamente se analizan los factores de ingreso en la Administración Local. El cuestionario de investigación planteaba la pregunta "cuál de los siguientes factores buscó al ingresar en la Administración", obteniéndose unos resultados que se entienden como los elementos determinantes que se buscaron por las personas para tomar la decisión de ingresar en la Administración Local (Gráfica 1). 


\section{Gráfica 1. Factores de ingreso en la Administración Local (primer factor)}

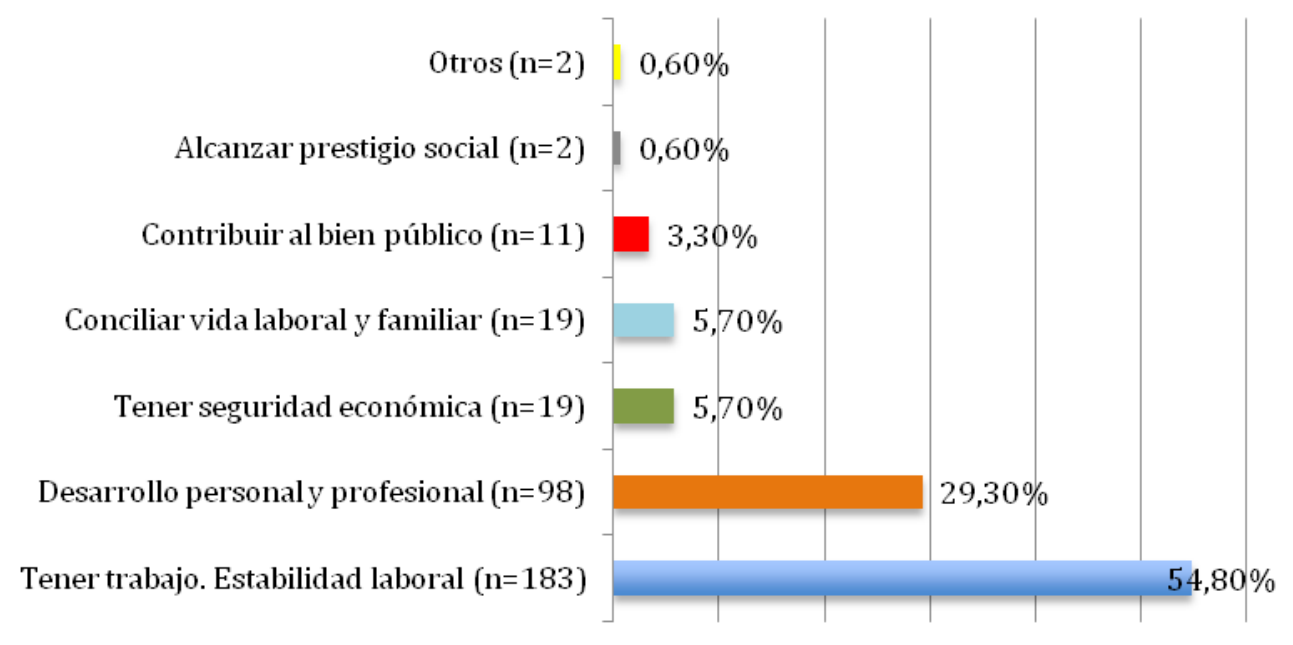

Fuente: Elaboración propia a partir del trabajo de campo.

De acuerdo con la Gráfica 1 se pueden obtener algunas conclusiones: a) la estabilidad laboral considerada como el tener trabajo y mantenerlo en el tiempo es, con gran diferencia, la primera razón que las personas buscan al decidir ingresar en la Administración Pública Local. Lo atestigua que el 54,8\% lo eligen como primer factor. Esta conclusión confirma la opinión muy asentada en la sociedad española al considerar este factor como uno de los más característicos que definen al empleo público; b) le sigue con un $29,3 \%$ el factor desarrollo personal y profesional.

Este resultado puede parecer sorprendente ya que rompe una lanza a favor del empleado público, en el sentido de que no renuncia a aspirar a una carrera profesional y confía en que la administración pueda permitirle desarrollarse profesionalmente; c) el resto de factores tienen una representación poco significativa en comparación con los factores comentados anteriormente, siendo paradójica la escasa importancia que se ha obtenido como primer factor a cuestiones que socialmente se le otorga mayor relevancia, como puede ser el conciliar la vida laboral y familiar o el tener seguridad económica, aspecto que pudiera estar relacionado con la estabilidad laboral; d) finalmente, los dos factores que menos importancia otorgan como factor que buscaron los empleados públicos son el contribuir al bien público $(3,3 \%)$ y alcanzar prestigio social $(0,6 \%)$. Ambos factores confirman el descrédito alcanzado por el empleo público y que la opinión pública confirma. Se considera que una forma de recuperar esa reputación consistiría en potenciar y desarrollar toda una estrategia de puesta en valor y despliegue de las habilidades personales y directivas, como una verdadera apuesta por el mérito y el rigor profesional.

Se continúa con el análisis de los resultados, destacando a continuación, las valoraciones que sobre las habilidades directivas se realizan por los empleados de la Administración Local, haciendo especial referencia a la inteligencia emocional (Cuadro 6). La inteligencia emocional como habilidad directiva es una habilidad que recibe una valoración que se puede calificar como intermedia dentro de un inventario de 22 habilidades directivas consideradas en la investigación. Se destaca una valoración media de la 
inteligencia emocional, que por el personal directivo alcanza un 3,75 sobre una escala de Likert de cinco puntos(cuando es el propio personal directivo el que se autovalora en relación a la habilidad directiva analizada), mientras que si es valorada por los empleados públicos que no ocupan puestos con personal a su cargo (respecto a su personal directivo), se reduce hasta el 3,14, aunque es preciso destacar la mayor desviación típica en las valoraciones de los subordinados (1,18 de media) frente a las de los directivos/ responsables ( 0,84 de media).

Lo que sí es concluyente es que se produce una mejor autovaloración de la inteligencia emocional por el personal directivo frente a la percepción de los subordinados. Como se observa en los resultados obtenidos en el Cuadro 6, la inteligencia emocional alcanza valoraciones muy parecidas a las que recibe otra megahabilidad como es el liderazgo, aunque muy por debajo de las habilidades que mejor valoración reciben, como son la capacidad de aprender, resolución de problemas y toma de decisiones y colaboración y trabajo en equipo. La diferencia en la valoración entre directivos y subordinados que se muestra en el Cuadro 6 confirma el hecho de que las personas en general, y directivos en particular, se "ven" mejor que como son percibidos y valorados por los demás, lo que ofrece enseñanzas interesantes para su discusión científica.

Cuadro 6. Valoración de las habilidades directivas

\begin{tabular}{|c|c|c|c|}
\hline Habilidad & \multicolumn{1}{c}{ Media P. } & \multicolumn{2}{c|}{ Media P. } \\
DIRECTIVO & SUBORDINADO & $\begin{array}{c}\text { Diferencia } \\
\text { media }\end{array}$ \\
\hline Capacidad de aprender & 4,28 & 3,5 & 0,78 \\
\hline Resolución de problemas y toma de decisiones & 4,14 & 3,42 & 0,72 \\
\hline Colaboración y trabajo en equipo & 4,11 & 3,39 & 0,72 \\
\hline Desarrollo personal & 3,97 & 3,1 & 0,87 \\
\hline Capacidad de comunicación & 3,92 & 3,5 & 0,42 \\
\hline Negociación & 3,91 & 3,19 & 0,72 \\
\hline Motivación & 3,88 & 3,21 & 0,67 \\
\hline Creatividad & 3,84 & 3,17 & 0,67 \\
\hline Liderazgo & 3,78 & 3,14 & 0,64 \\
\hline Resolución de conflictos & 3,76 & 3,26 & 0,5 \\
\hline Inteligencia emocional & 3,75 & 3,14 & 0,61 \\
\hline Asertividad & 3,71 & 3,25 & 0,46 \\
\hline Presentaciones en público & 3,70 & 3,33 & 0,37 \\
\hline Desarrollo de visión & 3,69 & 3,15 & 0,54 \\
\hline Delegación & 3,68 & 3,23 & 0,45 \\
\hline Gestión del tiempo & 3,65 & 3,24 & 0,41 \\
\hline Desarrollo de personas & 3,63 & 3,12 & 0,51 \\
\hline Optimización de recursos & 3,62 & 3,18 & 0,44 \\
\hline Gestión del estrés & 3,61 & 3,24 & 0,37 \\
\hline Gestión del talento & 3,58 & 3,01 & 0,57 \\
\hline Gestión de reuniones & 3,57 & 3,1 & 0,47 \\
\hline Coaching & 3,41 & 2,91 & 0,50 \\
\hline
\end{tabular}

Fuente: Elaboración propia a partir del trabajo de campo. 
A continuación se trata de profundizar, de forma global, en el análisis de las habilidades directivas pero desde una perspectiva diferente, es decir, desde su influencia en la entidad a través de determinadas variables organizacionales. La cuestión a dilucidar es si el empleado público "considera que una mayor y mejor consideración de las habilidades directivas y personales tendrían influencia positiva para la Administración”. Los datos obtenidos se presentan en el Cuadro 7, que muestra los estadísticos descriptivos (frecuencia, media y desviación típica), cuyos resultados revelan que los empleados públicos locales reconocen la existencia una alta relación e influencia en las habilidades directivas sobre determinadas variables organizacionales.

Cuadro 7. Influencia habilidades directivas en las variables organizacionales

\begin{tabular}{|c|c|c|c|}
\hline Variable & N & Media & Desv. típ. \\
\hline En la mejora de la eficacia y eficiencia & 354 & 4,47 & 0,76 \\
\hline En la motivación y satisfacción de los empleados públicos & 352 & 4,43 & 0,78 \\
\hline En los resultados alcanzados & 350 & 4,40 & 0,83 \\
\hline En el clima organizacional & 352 & 4,25 & 0,81 \\
\hline En la valoración y percepción de los ciudadanos & 349 & 4,06 & 0,98 \\
\hline En la selección de los empleados públicos & 347 & 3,99 & 1,03 \\
\hline
\end{tabular}

Fuente: Elaboración propia a partir del trabajo de campo.

De manera más detallada, de los resultados anteriores se pueden extraer las siguientes conclusiones: a) la influencia que los empleados públicos otorgan a las habilidades directivas sobre aspectos organizacionales es muy alta, como lo demuestra las medias obtenidas, todas, excepto una, por encima de 4 , sobre una escala de Likert de cinco puntos; b) resulta muy llamativo la gran influencia que los empleados públicos otorgan a las habilidades como responsable en la estrategia de mejora de la eficacia y eficiencia, así como en los resultados alcanzados por las organizaciones; c) igualmente, la mejora en la motivación y satisfacción de los empleados es un aspecto muy valorado como un resultado muy destacable en el deseado despliegue de las habilidades por parte del personal directivo, fundamentalmente; d) curiosamente, la única variable que se queda por debajo de 4, que aún siendo alta, es precisamente la influencia en la selección de los empleados públicos.

Este es un tema controvertido y a la vez sensible para el empleado público que, a priori, pudiera percibir como una pérdida de objetividad la introducción de elementos de difícil concreción como son las habilidades, cuya valoración pudiera estar más sometida a la subjetividad del tribunal calificador. Este es un tema de gran calado, que requiere un análisis muy específico, sobre qué sistemas de selección son los más adecuados a los objetivos de la entidad, y sobre todo, qué métodos permiten seleccionar a personas competentes, destacándose que la media obtenida $(3,99)$ identifica un verdadero interés en valorar que exclusivamente el conocimiento no es suficiente para garantizar la mejor selección, que las habilidades, el saber hacer, debería estar mucho más valorado en los procesos selectivos puesto que elevarían considerablemente la calidad de la selección de personal que, en definitiva, es el objetivo final, proporcionar a las organizaciones públicas empleados competentes y comprometidos. 
Así mismo, se ha utilizado el análisis de la varianza (Anova) para verificar la relación existente entre la inteligencia emocional respecto a determinadas variables sociodemográficas (sexo, estado civil, edad, formación, nivel salarial y formación en habilidades) obteniéndose resultados significativos que se exponen en el Cuadro 8.

Cuadro 8: Análisis de varianza (Anova): Inteligencia emocional - Perfil sociodemográfico

\begin{tabular}{|c|c|c|}
\hline SEXO & F & p-valor \\
\hline Inteligencia emocional (Directivos) & 0,71 & 0,586 \\
\hline Inteligencia emocional (Subordinados) & 0,87 & 0,485 \\
\hline ESTADO CIVIL & $\mathrm{F}$ & $\mathrm{p}$-valor \\
\hline Inteligencia emocional (Directivos) & 1,09 & 0,362 \\
\hline Inteligencia emocional (Subordinados) & 1,00 & 0,410 \\
\hline EDAD & $\mathrm{F}$ & $\mathrm{p}$-valor \\
\hline Inteligencia emocional (Directivos) & 0,74 & 0,570 \\
\hline Inteligencia emocional (Subordinados) & 2,54 & $0,041^{*}$ \\
\hline NIVEL FORMACION & $\mathrm{F}$ & $\mathrm{p}$-valor \\
\hline Inteligencia emocional (Directivos) & 1,51 & 0,202 \\
\hline Inteligencia emocional (Subordinados) & 0,50 & 0,738 \\
\hline NIVEL SALARIAL & $\mathrm{F}$ & $\mathrm{p}$-valor \\
\hline Inteligencia emocional (Directivos) & 0,82 & 0,515 \\
\hline Inteligencia emocional (Subordinados) & 1,27 & 0,282 \\
\hline FORMACIÓN HABILIDADES & $\mathrm{F}$ & $\mathrm{p}$-valor \\
\hline Inteligencia emocional (Directivos) & 2,26 & $0,065^{* *}$ \\
\hline
\end{tabular}

Fuente: Elaboración propia a partir del trabajo de campo.

De la relación edad/inteligencia emocional de los subordinados y formación habilidades/inteligencia emocional de los directivos se infieren conclusiones que permite valorar la importancia que la inteligencia emocional tienen para las organizaciones municipales, puesto que la no existencia de diferencias significativas ( 0,041 y 0,065 de valor " $p$ " asociado) muestra características a tener en cuenta. En este sentido, la edad y la formación en habilidades resulta ser una característica que dentro de los Ayuntamientos no ofrece diferencias en la valoración de la inteligencia emocional como habilidad directiva, para subordinados y directivos, respectivamente. En síntesis, estas dos conclusiones ofrecen posibilidades interesantes para la Administración Local que deberá considerar como variable estratégica en las decisiones a adoptar en el futuro, encaminadas a potenciar un capital humano deseoso de oportunidades y desarrollo personal y profesional.

En relación con determinadas variables organizacionales, en el Cuadro 9 se analiza la relación entre la inteligencia emocional con variables de gran trascendencia en gestión de las organizaciones públicas municipales (clima organizacional, motivación y satisfacción, mejora eficacia y eficiencia, selección empleados, etc.). 


\begin{tabular}{|c|c|c|c|c|c|c|}
\hline \multirow[t]{2}{*}{ Variable organizacional } & \multicolumn{3}{|c|}{$\begin{array}{l}\text { Inteligencia emocional } \\
\text { (Directivos) }\end{array}$} & \multicolumn{3}{|c|}{$\begin{array}{l}\text { Inteligencia emocional } \\
\text { (Subordinados) }\end{array}$} \\
\hline & Media & $\mathbf{F}$ & P-valor & Media & $\mathbf{F}$ & P-valor \\
\hline En el clima organizacional & 4,27 & 0,964 & 0,429 & 4,19 & 3,543 & $0,008 *$ \\
\hline En la mejora de la eficacia y eficiencia & 4,49 & 0,241 & 0,915 & 4,43 & 1,691 & 0,153 \\
\hline En la valoración y percepción de los ciudadanos & 4,10 & 0,546 & 0,715 & 4,05 & 1,511 & 0,200 \\
\hline En la motivación y satisfacción de los empleados & 4,40 & 2,449 & $0,049 *$ & 4,44 & 1,031 & 0,392 \\
\hline En la selección de los empleados públicos & 3,98 & 0,501 & 0,735 & 4,00 & 1,116 & 0,350 \\
\hline En los resultados alcanzados & 4,47 & 0,837 & 0,504 & 4,33 & 1,745 & 0,141 \\
\hline
\end{tabular}

* Significativo al 5\% ** Significativo al 10\%

Fuente: Elaboración propia a partir del trabajo de campo.

El análisis de la varianza (ANOVA) realizado muestra la incidencia que la valoración sobre la habilidad directiva inteligencia emocional tiene respecto a determinadas variables organizacionales. Se obtiene, en primer lugar, que no existen diferencias significativas en relación al sexo, tanto respecto a directivos como a subordinados. En cambio, en relación a otras variables, la significación desaparece en el estado civil y el nivel de formación, mientras que en la edad y nivel salarial, se obtiene que no existen diferencias significativas respecto a la consideración de la inteligencia emocional en los supuestos de subordinados y directivos, respectivamente.

Se considera que las variables analizadas confirman la percepción que para el directivo público tiene el desarrollo de la inteligencia emocional como habilidad directiva. El análisis ANOVA confirma una influencia significativa de la inteligencia emocional como habilidad directiva en la organización, especialmente en la motivación y satisfacción de los empleados públicos municipales. En cambio, respecto a la percepción de los subordinados los resultados muestra una influencia significativa en cuanto al clima organizacional. Esta circunstancia presenta a la inteligencia emocional como una habilidad transversal, por lo que las organizaciones, y las públicas en especial, deberán potenciarla adecuadamente.

\section{Conclusión}

La inteligencia emocional como habilidad directiva recibe de forma progresiva mayor importancia como lo corroboran las investigaciones realizadas y la apuesta por su desarrollo en muchas empresas y organizaciones. Las personas necesitan de esta habilidad para el desempeño eficiente de sus tareas y responsabilidades, por lo que su relevancia en la Administración Local en la Provincia de Córdoba es bastante clara.

Efectivamente, la inteligencia emocional se consolida como una habilidad directiva de gran trascendencia en empresas y administraciones públicas. No obstante, la importancia que tiene la inteligencia 
emocional no es valorada suficientemente, lo cual podría ser resultado de un insuficiente conocimiento de esta habilidad, si bien la difusión que está teniendo en los últimos años es ciertamente importante. Frente a otras habilidades que obtienen una valoración mucho más favorable en la Administración Local de la Provincia de Córdoba (Capacidad de aprender, Resolución de problemas y toma de decisiones y Cooperación y trabajo en equipo), la habilidad inteligencia emocional es valorada tanto por el personal directivo como subordinado, de manera, al menos, satisfactoria, lo cual ofrece una perspectiva favorable sobre la disposición del empelado público local por hacer frente adecuadamente a las múltiples exigencias que el servicio público demanda, especialmente debido al rol de servicio y adaptación a los ciudadanos, a los que en última instancia presta su servicio.

Por esto mismo, la inteligencia emocional puede convertirse en un factor de éxito para la propia administración local, es decir, contar con directivos y empleados emocionalmente inteligentes, capaces de adaptarse a los nuevos retos que exige un servicio público eficiente y eficaz, con gran adaptabilidad a los cambios y con una importante capacidad de comprender y atender a los ciudadanos. En definitiva, se trata de valorar y reconocer esta habilidad directiva como necesaria para un desempeño eficiente del trabajo, que se convierte en una estrategia de actuación y en un componente esencial en la línea de mejora continua de las organizaciones públicas locales más avanzadas.

La finalidad del presente artículo ha sido poder extraer una serie de conclusiones sobre una habilidad directiva, la inteligencia emocional, que cada vez tiene una mayor relevancia para las personas, por lo que viene siendo valorada de forma muy favorable por directivos y organizaciones en general, encontrando una valoración media superior cuando los directivos se autovaloran (respecto al desarrollo práctico de dicha habilidad), que cuando son valorados, en su aplicación, por sus subordinados, cuestión que parece razonable desde la perspectiva psicológica. Efectivamente, las personas tienden a valorarse mejor que como les ven los demás. Más aún en un ámbito tan especial como es el del trabajo, en el que aceptar y reconocer una actuación deficiente puede significar la desaprobación al desempeño realizado.

El perfil del empleado público local de la provincia de Córdoba viene determinado por las siguientes características: de género paritario hombre/mujer, con una edad de entre 46 y 55 años, de estado civil casado mayoritariamente; con formación universitaria, superando la mitad de la muestra seleccionada; con un nivel salarial que se sitúa entre los 1.000 y 2.000 euros (más del 65\%); mayoritariamente sin afiliación sindical y con formación específica en habilidades. El predominio del intervalo entre 46-55 años, implica una plantilla media madura, que provocará a medio plazo un fuerte proceso de jubilaciones, lo que supondrá un esfuerzo de planificación de recursos humanos muy notable al objeto de ir proporcionando los efectivos necesarios y adecuados.

Respecto a las características profesionales del empleado público local en la provincia de Córdoba vienen determinadas por una mayoría de personal funcionario (67,3\%), una alta representación de puestos que tienen personal a su cargo $(50,1 \%)$, siendo la mayoría de éstos $(85 \%)$ en un número de subordinados inferior a 25 empleados. Respecto al Grupo de clasificación actual, destacan dos grupos sobre el resto, el A1 y el I, como funcionario y personal laboral, respectivamente, que alcanza hasta el 26,5\%, y el C1 y III, que supone el $32 \%$ de la muestra.

A la vista de los resultados alcanzados, desde esta investigación se realizan una serie de recomendaciones a la hora de gestionar el capital humano en los ayuntamientos, que consistiría en la potenciación de las estrategias de formación, haciendo hincapié en los componentes de la inteligencia emocional descri- 
tos en la revisión de la literatura, como son la automotivación, el autoconocimiento y el autocontrol, que favorecerán un mejoramiento de la competencia personal en general. Este proceso se vería completado con la apuesta por seguir un proceso planificado que parta de las necesidades reales existentes y que no olvide evaluar los conocimientos y habilidades aprendidos y su posible aplicación al trabajo, así como el diseño de programas de formación de directivos con el objetivo de hacer de ellos impulsores del desarrollo de la inteligencia emocional como palanca de mejora organizacional.

Como futuras líneas de investigación sería interesante que se avanzase en la valoración esta habilidad directiva respecto a otras administraciones públicas (estatal, regional o universitaria) o incluso en relación al sector privado, para poder desarrollar propuestas más globales de actuación concretas que favorezcan el desarrollo y despliegue de la inteligencia emocional como habilidad directiva, entendida tanto globalmente como en cada uno de sus componentes.

\section{Referencias}

Alegre, Albert y Kenneth Levitt. 2014. The relation between emotional intelligence and transformational leadership: What do we really know? International Leadership Journal, 6 (2): 61-101.

Bar-On, Reuven. 1997. The bar-on emotional quotient inventory. Canada: Multi-Health Systems.

Batista, Judeira y Mabel Bermúdez. 2009. Inteligencia emocional y liderazgo femenino en cargos de dirección en organismos públicos. Revista REDHECS, 7 (4): 45-56.

Bisquerra, Rafael. 2003. Educación emocional y competencias para la vida. Revista de Investigación Educativa, 21(1): 7-43.

Blais, Ann-Renée y Bernard Sinclair-Dsegagné. 2002. Leadership in 3S`s: Skills, styles, situations. CIRANO. Centre interuniversitaire de recherche en analyse des organisations, BurgundyReport.

Cardona, Pablo y Nuria Chinchilla. 1999. Evaluación y desarrollo de las competencias directivas. Harvard Deusto Business Review, 89: 10-19.

Coggburn, Jerrell y Steven Hays. 2004. Innovations in local government human resource systems: Observations from several best practice locations. Public Administration Quarterly, 27(4): 433-455.

Collins, Chistopher y Kevin Clark. 2003. Strategic human resource practices, top management team social networks, and firm performance: The role of human resource practices in creating organizational competitive advantage. Academy of Management Journal, 46: 740-751. DOI: https://doi.org/10.2307/30040665 Cooper, Robert y Ayman Sawaf. 1988. La inteligencia emocional aplicada al liderazgo y las organizaciones. Colombia: Editorial Norma.

Del Pino, Rebeca y María de los Ángeles Aguilar. 2013. La inteligencia emocional como una herramienta de la gestión educativa para el liderazgo estudiantil. Cuadernos de Administración, 29 (50): 132-141.

Dolan, Simon. 2005. La inteligencia emocional, una habilidad para el éxito de los directivos globales. En Management knowledge innovation 2005. Cómo crear capacidades de aprendizaje y emprendizaje, pp. 24-28. Barcelona: ESADE.

García-Lombardía, Pilar, Pablo Cardona y María Chinchilla. 2001. Las competencias directivas más valoradas. Occasional Paper, 1/4.

Gardner, Howard. 1983. Frames of mind: The theory of multiple intelligences. Londres: William Heine- 
mann.

Goleman, Daniel. 1996. La inteligencia emocional. Barcelona: Editorial Kairós.

Goleman, Daniel. 1998a. La práctica de la inteligencia emocional. Barcelona: Editorial Kairós.

Goleman, Daniel. 1998b. What makes a leader? Harvard Bussiness Review, 76 (6): 93-102.

Goleman, Daniel. 1998c. La inteligencia emocional en la empresa. Barcelona: Editorial Planeta.

Goleman, Daniel. 2011. The brain and Emotional intelligence: New insights. Florence, MA: More Than-

Sound.

Goleman, Daniel y Cary Cherniss. 2005. Inteligencia emocional en el trabajo. Cómo seleccionar y mejorar la inteligencia emocional en individuos, grupos y organizaciones. Buenos Aires: Vergara.

González, Francisco, Sandra Sánchez y Tomás López-Guzmán. 2013. Influence of certain variables on organizacional commitment. A case study in the hotel sector of Córdoba. Tourismos. An International Multidisciplinary Journal of Tourism, 8 (2): 117-135.

Guillén, Laura y Elizabeth Florent-Treacy. 2011. Emotional intelligence and leadership effectiveness: The mediating influence of collaborative behaviors. INSEAD, Working Papers Collection, 23: 1-28.

Hopkins, Margaret, Deborah O’Neil y Helen Williams. 2007. Emotional intelligence and board governance: Leadership lessons from the public sector. Journal of Managerial Psychology, 22 (7): 683-700. DOI: https://doi.org/10.1108/02683940710820109

Instituto Nacional de Estadística. 2016a. INEbase / Demografía y población / Cifras de población y Censos demofiguras. Disponible en http://www.ine.es/jaxi/menu.do?type=pcaxis\&path=/t20/p321/ serie\&file $=$ pcaxis (14 de abril de 2016).

Instituto Nacional de Estadística. 2016b. Informe del mercado de trabajo de Córdoba. Servicio Público de Empleo Estatal. Madrid.

Kim, Andrea y Choonwoo Lee. 2012. How does HRM enhance strategic capabilities? Evidence from the Korean management consulting industry. The International Journal of Human Resource Management, 23: 126-146. DOI: https://doi.org/10.1080/09585192.2011.561247

Lee, Feng-Hui, Tzai-Zang Lee y Wann-Yih Wu. 2010. The relationship between human resource management practices, business strategy and firm performance: Evidence from steel industry in Taiwan. The International Journal of Human Resource Management, 21: 1351-1372. DOI: https://doi.org/10.1080/0 9585192.2010.488428

López-Boudet, Roberto y Ángel Martínez-Vázquez.2014. Inteligencia emocional y “La ventana del líder” en los directivos turísticos. Ingeniería Industrial, 35(2): 229-239.

Madrigal, Berta. 2009. Habilidades directivas. México: McGraw-Hill.

Ministerio de Hacienda y Administraciones Públicas. 2015. Boletín estadístico del personal al servicio de las administraciones públicas. Registro Central de Personal, Enero 2014. Dirección General de Modernización Administrativa, Procedimientos e Impulso de la Administración Electrónica. Madrid.

Robina, Rafael. 2002. Condicionantes sociolaborales de los empleados públicos. Motivación y satisfacción laboral en la Administración Regional Extremeña. Tesis doctoral. Universidad de Extremadura, Cáceres.

Ryback, David. 1998. Trabaje con su inteligencia. Los factores emocionales al servicio de la gestión institucional y el liderazgo efectivo. Madrid: Edaf.

Ryback, David. 2005. EQ: Trabaje con su inteligencia emocional. Madrid: Edaf. 
Salovey, Peter y John Mayer. 1990. Emotional intelligence. Imagination, Cognition and Personality, 9: 185-211. DOI: https://doi.org/10.2190/DUGG-P24E-52WK-6CDG

Sanz, Raquel y Ramón Sánchez. 2002. Enfoque universalista de recursos humanos. Estudio empírico de sus efectos sobre los resultados de la empresa. Revista Europea de Dirección y Economía de la Empresa, 11(1): 163-182.

Serrano, José. y María Barba. 2012. Human resource management in local governments. Cuadernos de Gestión, 12(2): 149-168.

Sternberg, Robert. 1997. Inteligencia exitosa. Barcelona: Paidós.

Wang, Jiankang y Jiuling Xiao. 2009. Knowledge management audit framework and methodology based on processes. Journal of Technology Management in China, 4(3): 239-249. DOI: https://doi. org/10.1108/17468770911013546

Weisinger, Hendrie. 2001. La inteligenciaemocional en el trabajo. Buenos Aires: Vergara.

Wright, Patrick, Timothy Gardner y Lisa Moynihan. 2003. The impact of HR practices on the performance of the business. Human Resource Management Journal, 13(3): 21-36. DOI: https://doi. org/10.1111/j.1748-8583.2003.tb00096.x

Zárate, Rodrigo y Sergio Matviuk. 2012. La inteligencia emocional de los líderes latinoamericanos: comparación entre Chile y Colombia. REAL-Revista de Estudios Avanzados de Liderazgo, 1 (1): 43-50. 